\title{
Socle theory for Leavitt path algebras of arbitrary graphs
}

\section{Gonzalo Aranda Pino, Dolores Martín Barquero, Cándido Martín González and Mercedes Siles Molina}

\begin{abstract}
The main aim of the paper is to give a socle theory for Leavitt path algebras of arbitrary graphs. We use both the desingularization process and combinatorial methods to study Morita invariant properties concerning the socle and to characterize it, respectively. Leavitt path algebras with nonzero socle are described as those which have line points, and it is shown that the line points generate the socle of a Leavitt path algebra. A concrete description of the socle of a Leavitt path algebra is obtained: it is a direct sum of matrix rings (of finite or infinite size) over the base field.

New proofs of the Graded Uniqueness and of the Cuntz-Krieger Uniqueness Theorems are given, by using very different means.
\end{abstract}

\section{Introduction}

Leavitt path algebras of row-finite graphs have been recently introduced in [1] and [9]. They have become a subject of significant interest, both for algebraists and for analysts working in $\mathrm{C}^{*}$-algebras. The Cuntz-Krieger algebras $C^{*}(E)$ (the $\mathrm{C}^{*}$-algebra counterpart of these Leavitt path algebras) are described in [27]. The algebraic and analytic theories, while sharing some striking similarities, present some remarkable differences, as was shown for instance in the "Workshop on Graph Algebras" held at the University of Málaga (see [11]), and more deeply in the subsequent enlightening work by Tomforde [31].

For a field $K$, the algebras $L_{K}(E)$ are natural generalizations of the algebras investigated by Leavitt in [26], and are a specific type of path $K$ algebras associated to a graph $E$ (modulo certain relations). The family of

2000 Mathematics Subject Classification: $16 \mathrm{D} 70$.

Keywords: Leavitt path algebra, graph $\mathrm{C}^{*}$-algebra, socle, arbitrary graph, minimal left ideal. 
algebras which can be realized as the Leavitt path algebra of a graph includes matrix rings $\mathbb{M}_{n}(K)$ for $n \in \mathbb{N} \cup\{\infty\}$ (where $\mathbb{M}_{\infty}(K)$ denotes matrices of countable size with only a finite number of nonzero entries), the Toeplitz algebra, the Laurent polynomial ring $K\left[x, x^{-1}\right]$, and the classical Leavitt algebras $L(1, n)$ for $n \geq 2$. Constructions such as direct sums, direct limits and matrices over the previous examples can be also realized in this setting. But, in addition to the fact that these structures indeed contain many wellknown algebras, one of the main interests in their study is the comfortable pictorial representation that their corresponding graphs provide.

The development of the theory of Leavitt path algebras (as well as that of their analytic sisters, the graph $\mathrm{C}^{*}$-algebras) has had several different stages as far as questions of cardinality of the graphs are concerned. At first, in the $\mathrm{C}^{*}$-case, only finite graphs (represented by matrices) were considered: Cuntz [16] constructed and investigated the $\mathrm{C}^{*}$-algebras $\mathcal{O}_{n}$ (nowadays called the Cuntz algebras), showing, among other things, that each $\mathcal{O}_{n}$ is (algebraically) simple. Soon after the appearance of [16], Cuntz and Krieger [17] described the significantly more general notion of the $\mathrm{C}^{*}$-algebra of a (finite) matrix $A$, denoted $\mathcal{O}_{A}$. Among this class of $\mathrm{C}^{*}$-algebras one can find, for certain finite graphs $E$, the Cuntz-Krieger algebras $C^{*}(E)$, defined originally in [24]. The algebraic counterpart of these finite Cuntz-Krieger algebras was considered in [7].

The second step was to consider possibly infinite but countable row-finite graphs (that is, graphs with a countable number of vertices and edges which satisfy that a vertex in the graph emits at most a finite number of edges). This was first done in the analytic setting (see [15, 27, 28] among others), while the seminal results on Leavitt path algebras of row-finite graphs appeared in [1] and [9], so starting a flurry of activity. In both situations the classification of simple ([1]) and purely infinite simple ([2]) structures was carried out in terms of properties of the graph. In the analytic situation, the gauge invariant ideals were determined; in the algebraic one, the graded ideals were described. In addition, several other ring properties were studied in the case of row-finite Leavitt path algebras, such as being exchange [10], finite dimensional [5], noetherian [6], semisimple [4] or having stable rank $[10,8]$. It has been also shown that the Leavitt path algebra $L_{K}(E)$ of a graph $E$ and the path algebra $K E$ associated to the same graph are closely enough: $L_{K}(E)$ is an algebra of right quotients of $K E$ (see [29]).

Apart from the very recent paper [22], by K. R. Goodearl, where he has introduced Leavitt path algebras of uncountable directed graphs, the following breakthrough was to remove the hypothesis of row-finiteness in the underlying graphs. Once more this was first done for the case of graph $\mathrm{C}^{*}$ algebras $C^{*}(E)$ (see for instance $[19,14,18]$ ) and afterwards for Leavitt path 
algebras in $[3,31]$. In both, the analytic and the algebraic cases, very often the validity of the results for these not necessarily row-finite graphs requires coming up with totally different proofs to those given for the row-finite case. Sometimes, helpful shortcuts such as the desingularization process are at hand and sometimes the proofs must be reinvented. In this paper we face both situations.

Concretely, the aim of this article is to extend the theory of the socle of a Leavitt path algebra (considered in [12] for row-finite graphs) to (countable) not necessarily row-finite graphs. Specifically, we determine the structure of minimal left ideals of Leavitt path algebras of arbitrary graphs and we scrutinize the nature of the socle of a Leavitt path algebra of an arbitrary graph in two different ways: first, by singling out the set of vertices that generate the socle as a two-sided ideal (graph description) and secondly, by unveiling the internal algebraic structure of it (algebraic description).

It is worth mentioning that these results on the socle were successfully applied for the row-finite case in [4] in order to completely classify the semisimple/locally noetherian/locally artinian Leavitt path algebras. Hence, this extension of [12] to arbitrary graphs could potentially led to achievements analogous to [4] but for arbitrary graphs.

The article is organized as follows. The first section includes the basic definitions and examples that will be used throughout. In addition, we describe several basic results and relations between the path algebra and the Leavitt path algebra of an arbitrary graph.

In Section 2, a first approach to the study of the socle of a Leavitt path algebra of an arbitrary graph via the desingularization process is made. We relate the "line point" vertices of a graph (these are the vertices that generate the socle as an ideal) to that of its desingularization. This allows us to establish, in Corollary 2.5, further socle-related connections between the Leavitt path algebra of an arbitrary graph $E$ and the Leavitt path algebra of its desingularized (row-finite) graph $F$. These are: $L_{K}(E)$ has nonzero socle if and only if $L_{K}(F)$ has nonzero socle and $L_{K}(E)$ coincides with its socle if and only if $L_{K}(F)$ coincides with its socle.

The action of the multiplication algebra is considered in Section 3. This is a valuable tool that allowed us to shorten the proofs of the Graded Uniqueness Theorem and the Cuntz-Krieger Uniqueness Theorem for Leavitt path algebras of arbitrary graphs given in [31]. What is more, this tool allowed us to weaken, in Theorem 3.7, the set of hypotheses of the aforementioned Cuntz-Krieger Uniqueness Theorem to a level that was useful for instance in [3, Proof of Proposition 5.1]. These uniqueness theorems allow us to obtain that for a graph, the socle of the Leavitt path algebra can be seen inside the socle of the corresponding graph $\mathrm{C}^{*}$-algebra. 
The study of minimal left ideals of Leavitt path algebras of arbitrary graphs is carried out in Section 4. There are two phases for this: first, only principal left ideals generated by vertices are considered; afterwards, principal left ideals generated by an arbitrary element of $L_{K}(E)$ are determined. One of the key tools in [12] for this same enterprise in the row-finite case was the possibility to see the algebra $L_{K}(E)$ as a certain direct limit of algebras associated to finite complete subgraphs. However, this construction is no longer available in the general case of arbitrary graphs, so a completely different approach, of a more combinatorial nature, is needed here.

Having paved the way, the natural subsequent and final step is taken in Section 5, where the socle of a Leavitt path algebra of an arbitrary graph is determined. Thus, the graph description of the socle is given in Theorem 5.2 and is the following: the socle of a Leavitt path algebra is the two-sided ideal generated by the vertices of the graph whose trees (that is, the vertices which follow them in the graph) do not contain cycles nor bifurcations (i.e., the socle is the two-sided ideal generated by all line points). On the other hand, the algebraic description of the socle is given in Theorem 5.6: the socle of a Leavitt path algebra is a direct sum of full matrix algebras over the field $K$ of either finite or countably infinite size.

\section{Path algebras and Leavitt path algebras of arbitrary graphs}

A (directed) graph $E=\left(E^{0}, E^{1}, r, s\right)$ consists of two countable sets $E^{0}$ and $E^{1}$, and maps $r, s: E^{1} \rightarrow E^{0}$. The elements of $E^{0}$ are called vertices and the elements of $E^{1}$ edges. If for a vertex $v$, the set $s^{-1}(v)$ is finite, then the graph is called row-finite. If $E^{0}$ is finite then, by the row-finite hypothesis, $E^{1}$ must necessarily be finite as well; in this case we say simply that $E$ is finite. A vertex is called a sink if it does not emit edges, and a source if it does not receive edges. A vertex $v$ such that $\left|s^{-1}(v)\right|=\infty$ is called an infinite emitter. Following [31], if $v$ is either a sink or an infinite emitter, we call it a singular vertex. If $v$ is not a singular vertex, we will say that it is a regular vertex. A path $\mu$ in a graph $E$ is a sequence of edges $\mu=e_{1} \ldots e_{n}$ such that $r\left(e_{i}\right)=s\left(e_{i+1}\right)$ for $i=1, \ldots, n-1$. In this case, $s(\mu):=s\left(e_{1}\right)$ is the source of $\mu, r(\mu):=r\left(e_{n}\right)$ is the range of $\mu$, and $n$ is the length of $\mu$, i.e, $l(\mu)=n$. We denote by $\mu^{0}$ the set of its vertices, that is: $\mu^{0}=\left\{s\left(e_{1}\right), r\left(e_{i}\right) \mid i=1, \ldots, n\right\}$.

An edge $e$ is an exit for a path $\mu=e_{1} \ldots e_{n}$ if there exists $i$ such that $s(e)=s\left(e_{i}\right)$ and $e \neq e_{i}$. If $\mu$ is a path in $E$, and if $v=s(\mu)=r(\mu)$, then $\mu$ is called a closed path based at $v$. Let $C P(v)$ denote the set of all closed paths based at $v$. If $s(\mu)=r(\mu)$ and $s\left(e_{i}\right) \neq s\left(e_{j}\right)$ for every $i \neq j$, then $\mu$ is called a cycle. 
We say that a graph $E$ satisfies Condition (L) if every cycle in $E$ has an exit. For $n \geq 2$ we define $E^{n}$ to be the set of paths of length $n$, and $E^{*}=\bigcup_{n \geq 0} E^{n}$ the set of all paths.

The set $T(v)=\left\{w \in E^{0} \mid v \geq w\right\}$ is the tree of $v$, that is, the set of all the vertices in the graph $E$ which follow $v(v \geq w$ means that there is a path $\mu$ with $s(\mu)=v$ and $r(\mu)=w)$. We will denote it by $T_{E}(v)$ when it is necessary to emphasize the dependence on the graph $E$.

Now let $K$ be a field and let $K E$ denote the $K$-vector space which has as a basis the set of paths. It is possible to define an algebra structure on $K E$ as follows: for any two paths $\mu=e_{1} \ldots e_{m}, \nu=f_{1} \ldots f_{n}$, we define $\mu \nu$ as zero if $r(\mu) \neq s(\nu)$ and as the path $e_{1} \ldots e_{m} f_{1} \ldots f_{n}$ otherwise. This $K$-algebra is called the path algebra of $E$ over $K$.

For a field $K$ and a graph $E$, the Leavitt path $K$-algebra $L_{K}(E)$ is defined as the $K$-algebra generated by a set $\left\{v \mid v \in E^{0}\right\}$ of pairwise orthogonal idempotents, together with a set of variables $\left\{e, e^{*} \mid e \in E^{1}\right\}$, which satisfy the following relations:

(1) $s(e) e=e r(e)=e$ for all $e \in E^{1}$.

(2) $r(e) e^{*}=e^{*} s(e)=e^{*}$ for all $e \in E^{1}$.

(3) $e^{*} e^{\prime}=\delta_{e, e^{\prime}} r(e)$ for all $e, e^{\prime} \in E^{1}$.

(4) $v=\sum_{\left\{e \in E^{1} \mid s(e)=v\right\}} e e^{*}$ for every regular vertex $v \in E^{0}$.

The elements of $E^{1}$ are called (real) edges, while for $e \in E^{1}$ we call $e^{*}$ a ghost edge. The set $\left\{e^{*} \mid e \in E^{1}\right\}$ will be denoted by $\left(E^{1}\right)^{*}$. We let $r\left(e^{*}\right)$ denote $s(e)$, and we let $s\left(e^{*}\right)$ denote $r(e)$. If $\mu=e_{1} \ldots e_{n}$ is a path, then we denote by $\mu^{*}$ the element $e_{n}^{*} \ldots e_{1}^{*}$ of $L_{K}(E)$.

Formally speaking we can say that $L_{K}(E)$ is the quotient of the free associative algebra generated by $E^{0} \cup E^{1} \cup\left(E^{1}\right)^{*}$ modulo the ideal induced by the identities (1)-(4). Hence the universal property of the free associative algebra jointly with that of the quotient algebra can be used to construct homomorphisms with domain $L_{K}(E)$.

We will recall some facts that will be used freely along the paper.

The Leavitt path $K$-algebra $L_{K}(E)$ is spanned as a $K$-vector space by $\left\{p q^{*} \mid p, q\right.$ are paths in $\left.E\right\}$ (see [31, Lemma 3.1]). Moreover, $L_{K}(E)$ has a natural $\mathbb{Z}$-grading (see [31, Section 3.3]): for each $n \in \mathbb{Z}$, the degree $n$ component $L_{K}(E)_{n}$ is spanned by elements of the form $p q^{*}$ where $l(p)-l(q)=n$.

The set of homogeneous elements is $\bigcup_{n \in \mathbb{Z}} L_{K}(E)_{n}$, and an element $x \in$ $L_{K}(E)_{n}$ is said to be $n$-homogeneous or homogeneous of degree $n$, denoted by $\operatorname{deg}(x)=n$.

The $K$-linear extension of the assignment $p q^{*} \mapsto q p^{*}$ (for $p, q$ paths in $E$ ) yields an involution on $L_{K}(E)$, which we denote simply as * . Clearly $\left(L_{K}(E)_{n}\right)^{*}=L_{K}(E)_{-n}$ for all $n \in \mathbb{Z}$. 
Examples 1.1. By considering some basic configurations one can realize many algebras as the Leavitt path algebra of some graph. Thus, for instance, the ring of Laurent polynomials $K\left[x, x^{-1}\right]$ is the Leavitt path algebra of the graph

Matrix algebras $M_{n}(K)$ can be achieved by considering a line graph with $n$ vertices and $n-1$ edges

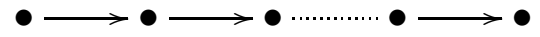

Classical Leavitt algebras $L(1, n)$ for $n \geq 2$ are obtained as $L\left(R_{n}\right)$, where $R_{n}$ is the rose with $n$ petals graph

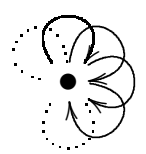

Of course, combinations of the previous examples are possible. For example, the Leavitt path algebra of the graph

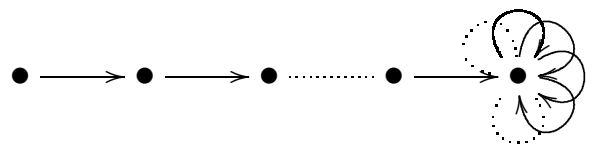

is $M_{n}(L(1, m))$, where $n$ denotes the number of vertices in the graph and $m$ denotes the number of loops. In addition, the algebraic counterpart of the Toeplitz algebra $T$ is the Leavitt path algebra of the graph $E$ having one loop and one exit

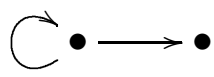

There exists a natural inclusion of the path algebra $K E$ into the Leavitt path algebra $L_{K}(E)$ sending vertices to vertices and edges to edges. We will use this monomorphism without any explicit mention to it. Moreover, this natural monomorphism from the path algebra $K E$ into the Leavitt path algebra $L_{K}(E)$ is graded, hence $K E$ is a $\mathbb{Z}$-graded subalgebra of $L_{K}(E)$.

We will revisit some basic results on the path algebra $K E$ and on the Leavitt path algebra $L_{K}(E)$ for an arbitrary graph $E$. Given that the only difference between the definition of the Leavitt path algebra of a row-finite graph and of an arbitrary graph is the non-existence of a CK2 relation ((4) in the definition) at infinite emitters, it is perhaps not surprising that many of the results that hold for the row-finite case still hold in this more general situation. In particular, by rereading the result in [29, Lemma 1.1] we get that the following still holds in this more general situation. 
Lemma 1.2. Let $E$ be an arbitrary graph. Any set of different paths is $K$-linearly independent.

The same can be said about [29, Proposition 2.1]:

Proposition 1.3. Let $E$ be an arbitrary graph. Then the path algebra $K E$ is semiprime if and only if for every path $\mu$ there exists a path $\nu$ such that $s(\nu)=r(\mu)$ and $r(\nu)=s(\mu)$.

Also, following the proof of [29, Proposition 2.2] and applying [13, Lemma 1.12] we have:

Proposition 1.4. For an arbitrary graph $E$ and any field $K$, the Leavitt path algebra $L_{K}(E)$ is an algebra of right quotients of the path algebra $K E$, equivalently, it is a $\mathbb{Z}$-graded algebra of right quotients of $K E$.

The following result was stated for row-finite graphs in [1, Theorem 3.11]. We include here a proof for arbitrary graphs.

Lemma 1.5. Let $E$ be an arbitrary graph. Let $v$ be a vertex in $E^{0}$ such that there exists a cycle without exits $c$ based at $v$. Then:

$$
v L_{K}(E) v=\left\{\sum_{i=-m}^{n} k_{i} c^{i} \mid k_{i} \in K ; m, n \in \mathbb{N}\right\} \cong K\left[x, x^{-1}\right],
$$

where $\cong$ denotes a graded isomorphism of $K$-algebras, and considering (by abuse of notation) $c^{0}=w$ and $c^{-t}=\left(c^{*}\right)^{t}$, for any $t \geq 1$.

Proof. First, it is easy to see that if $c=e_{1} \ldots e_{n}$ is a cycle without exits based at $v$ and $u \in T(v)$, then $s(f)=s(g)=u$, for $f, g \in E^{1}$, implies $f=g$. Moreover, if $r(h)=r(j)=w \in T(v)$, with $h, j \in E^{1}$, and $s(h), s(j) \in T(v)$ then $h=j$. We have also that if $\mu \in E^{*}$ and $s(\mu)=u \in T(v)$ then there exists $k \in \mathbb{N}^{*}, 1 \leq k \leq n$ verifying $\mu=e_{k} \mu^{\prime}$ and $s\left(e_{k}\right)=u$.

Let $x \in v L_{K}(E) v$ be given by $x=\sum_{i=1}^{p} k_{i} \alpha_{i} \beta_{i}^{*}+\delta v$, with $s\left(\alpha_{i}\right)=r\left(\beta_{i}^{*}\right)=$ $s\left(\beta_{i}\right)=v$ and $\alpha_{i}, \beta_{i} \in E^{*}$. Consider $A=\left\{\alpha \in E^{*}: s(\alpha)=v\right\}$; we prove now that if $\alpha \in A, \operatorname{deg}(\alpha)=m n+q, m, q \in \mathbb{N}$ with $0 \leq q<n$, then $\alpha=c^{m} e_{1} \ldots e_{q}$. We proceed by induction on $\operatorname{deg}(\alpha)$. If $\operatorname{deg}(\alpha)=1$ and $s(\alpha)=s\left(e_{1}\right)$ then $\alpha=e_{1}$. Suppose now that the result holds for any $\beta \in A$ with $\operatorname{deg}(\beta) \leq s n+t$ and consider any $\alpha \in A$, with $\operatorname{deg}(\alpha)=s n+t+1$. We can write $\alpha=\alpha^{\prime} f$ with $\alpha^{\prime} \in A, f \in E^{1}$ and $\operatorname{deg}\left(\alpha^{\prime}\right)=s n+t$, so by the induction hypothesis $\alpha^{\prime}=c^{s} e_{1} \ldots e_{t}$. Since $s(f)=r\left(e_{t}\right)=s\left(e_{t+1}\right)$ implies $f=e_{t+1}$, then $\alpha=\alpha^{\prime} f=c^{s} e_{1} \ldots e_{t+1}$.

We shall show that the elements $\alpha_{i} \beta_{i}^{*}$ are in the desired form, i.e., $c^{d}$ with $d \in \mathbb{Z}$. Indeed, if $\operatorname{deg}\left(\alpha_{i}\right)=\operatorname{deg}\left(\beta_{i}\right)$ and $\alpha_{i} \beta_{i}^{*} \neq 0$, we have $\alpha_{i} \beta_{i}^{*}=$ 
$c^{p} e_{1} \ldots e_{k} e_{k}^{*} \ldots e_{1}^{*} c^{-p}=v$ by (4). On the other hand $\operatorname{deg}\left(\alpha_{i}\right)>\operatorname{deg}\left(\beta_{i}\right)$ and $\alpha_{i} \beta_{i}^{*} \neq 0$ imply $\alpha_{i} \beta_{i}^{*}=c^{d+q} e_{1} \ldots e_{k} e_{k}^{*} \ldots e_{1}^{*} c^{-q}=c^{d}, d \in \mathbb{N}^{*}$. In a similar way, from $\operatorname{deg}\left(\alpha_{i}\right)<\operatorname{deg}\left(\beta_{i}\right)$ and $\alpha_{i} \beta_{i}^{*} \neq 0$ it follows that $\alpha_{i} \beta_{i}^{*}=$ $c^{q} e_{1} \ldots e_{k} e_{k}^{*} \ldots e_{1}^{*} c^{-q-d}=c^{-d}, d \in \mathbb{N}^{*}$. Define $\varphi: K\left[x, x^{-1}\right] \rightarrow L_{K}(E)$ by $\varphi(1)=v, \varphi(x)=c$ and $\varphi\left(x^{-1}\right)=c^{*}$. It is a straightforward routine to check that $\varphi$ is a graded monomorphism with image $v L_{K}(E) v$, so that $v L_{K}(E) v$ is graded isomorphic to $K\left[x, x^{-1}\right]$ as a graded $K$-algebra.

\section{Desingularization, Morita equivalence and socle}

Given an arbitrary graph $E$, one can associate a row-finite graph $F$, called a desingularization of $E$, such that $L_{K}(E)$ is Morita equivalent to $L_{K}(F)$ as rings with local units [3, Theorem 5.2]. The process of building the graph $F$ out of $E$ is described in $[18,3]$ and it essentially consists, as the name suggests, on conveniently removing the singular vertices of $E$. We briefly recall the process here for the reader's convenience:

If $v_{0}$ is a sink in $E$, then by adding a tail at $v_{0}$ we mean attaching a graph of the form

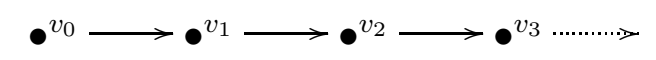

to $E$ at $v_{0}$. If $v_{0}$ is an infinite emitter in $E$, then by adding a tail at $v_{0}$ we mean performing the following process: we first list the edges $e_{1}, e_{2}, e_{3}, \ldots$ of $s^{-1}\left(v_{0}\right)$, then we add a tail to $E$ at $v_{0}$ of the following form

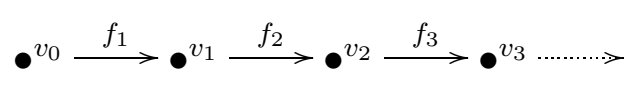

We remove the edges in $s^{-1}\left(v_{0}\right)$, and for every $e_{j} \in s^{-1}\left(v_{0}\right)$ we draw an edge $g_{j}$ from $v_{j-1}$ to $r\left(e_{j}\right)$.

If $E$ is a directed graph, then a desingularization of $E$ is a graph $F$ formed by adding a tail to every sink and every infinite emitter of $E$ in the fashion above. Several basic examples of desingularized graphs are found in [3, Examples 5.1, 5.2 and 5.3].

When extending results to Leavitt path algebras of arbitrary graphs two main philosophies have been followed. The obvious one consists on just reproving the results for arbitrary graphs with some ad hoc methods, while the second approach uses the aforementioned desingularization construction which allows us to transfer, via a Morita equivalence, results from the arbitrary graph setting to the row-finite situation.

In this section, we will obtain information about the socle of an arbitrary Leavitt path algebra $L_{K}(E)$ out of the information provided by the socle of the Leavitt path algebra of its desingularization $L_{K}(F)$. 
A vertex $v$ in $E^{0}$ is a bifurcation (or there is a bifurcation at $v$ ) if $s^{-1}(v)$ has at least two elements. A vertex $u$ in $E^{0}$ will be called a line point if there are neither bifurcations nor cycles at any vertex $w \in T(u)$. We will denote by $P_{l}(E)$ the set of all line points in $E^{0}$.

As we will show, the set of line points plays a crucial role in the description of the socle of a Leavitt path algebra of an arbitrary graph $E$. The next results analyze the relation between the line points of $E$ and those of the desingularized graph $F$.

Proposition 2.1. Let $E$ be an arbitrary graph and $F$ any desingularization of $E$. Then

(1) $P_{l}(E)=P_{l}(F) \cap E^{0}$.

(2) $P_{l}(E) \neq \emptyset$ if and only if $P_{l}(F) \neq \emptyset$.

Proof. (1). Suppose that $v \in P_{l}(E)$. Then $T_{E}(v)$ does not contain bifurcations nor cycles in $E$; in particular, it does not contain infinite emitters in $E$. Therefore, no edges are added at any vertex of $T_{E}(v)$ in the desingularization process unless $T_{E}(v)$ contains a (necessarily unique) sink $w$, in which case an infinite tail of the form

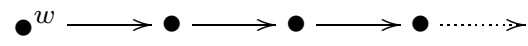

has been attached at $w$. In other words, $T_{F}(v)$ does not contain bifurcations nor cycles in $F$ either, that is, $v \in P_{l}(F)$.

To see the converse containment, take $v \in P_{l}(F) \cap E^{0}$. Then $T_{F}(v)$ does not contain bifurcations nor cycles in $F$. Note that, by construction, neither vertex of $T_{F}(v)$ is a sink nor an infinite emitter. All this shows that there exists a countable family of vertices $\left\{v_{i}\right\}_{i=0}^{\infty}$ and edges $\left\{e_{i}\right\}_{i=0}^{\infty}$ of $F$ such that $v_{0}=v, s_{F}^{-1}\left(v_{i}\right)=\left\{e_{i}\right\}$ for all $i$ and $T_{F}(v)=\left\{v_{i}\right\}_{i=0}^{\infty}$.

Since $v \in E^{0}$ we have two situations. First, if every $v_{i}$ was already in $E$, then from the way the graph $F$ is constructed we conclude that $T_{E}(v)=$ $\left\{v_{i}\right\}_{i=0}^{\infty}$, and $s_{E}^{-1}\left(v_{i}\right)=\left\{e_{i}\right\}$ for all $i$. Otherwise, there exists $j \geq 0$, such that $v_{j}$ is a sink in $E^{0}, T_{E}(v)=\left\{v_{i}\right\}_{i=0}^{j}$, and $s_{E}^{-1}\left(v_{i}\right)=\left\{e_{i}\right\}$ for all $i \leq j$. In both cases $v \in P_{l}(F)$ implies that $v \in P_{l}(E)$.

(2). If $P_{l}(E) \neq \emptyset$, then $P_{l}(F) \neq \emptyset$ by (1). Suppose now that $v \in P_{l}(F)$. Again, if $v \in E^{0}$, then $v \in P_{l}(E)$ by (1). Otherwise, if $v$ is a vertex which was not originally if $E$, then it cannot be a vertex in $\left\{v_{i}\right\}_{i \geq 1}$ of any new infinite tail of the form

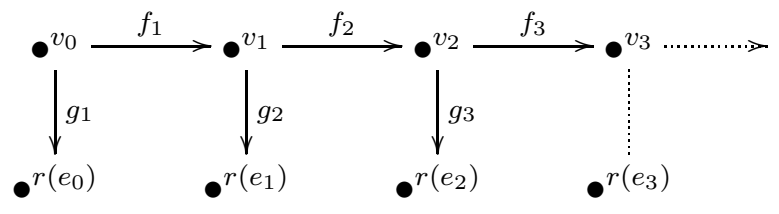


as the trees of all vertices $\left\{v_{i}\right\}_{i \geq 0}$ of these configurations necessarily have bifurcations. Therefore, $v$ is a vertex of an infinite tail of $F$ which was introduced at a sink $z$ in $E$, but then clearly $z \in P_{l}(E)$. So that $P_{l}(E) \neq \emptyset$ in this case too.

One of the main results of [3] was [3, Theorem 5.2]. There, the authors proved that if $E$ is an arbitrary graph, then $L_{K}(E)$ is Morita equivalent to $L_{K}(F)$ for any desingularization $F$ of $E$. We are going to exploit that fact in this section. First, we recall the notion of Morita equivalence for idempotent rings (a ring $R$ is said to be idempotent if $R^{2}=R$ ). Note that since Leavitt path algebras have local units, they are idempotent rings.

Let $R$ and $S$ be two rings, ${ }_{R} N_{S}$ and ${ }_{S} M_{R}$ two bimodules and $(-,-)$ : $N \times M \rightarrow R,[-,-]: M \times N \rightarrow S$ two maps. Then the following conditions are equivalent:

(i) $\left(\begin{array}{cc}R & N \\ M & S\end{array}\right)$ is a ring with componentwise sum and product given by:

$$
\left(\begin{array}{cc}
r_{1} & n_{1} \\
m_{1} & s_{1}
\end{array}\right)\left(\begin{array}{cc}
r_{2} & n_{2} \\
m_{2} & s_{2}
\end{array}\right)=\left(\begin{array}{cc}
r_{1} r_{2}+\left(n_{1}, m_{2}\right) & r_{1} n_{2}+n_{1} s_{2} \\
m_{1} r_{2}+s_{1} m_{2} & {\left[m_{1}, n_{2}\right]+s_{1} s_{2}}
\end{array}\right)
$$

(ii) $[-,-]$ is $S$-bilinear and $R$-balanced, $(-,-)$ is $R$-bilinear and $S$-balanced and the following associativity conditions hold:

$$
(n, m) n^{\prime}=n\left[m, n^{\prime}\right] \quad \text { and } \quad[m, n] m^{\prime}=m\left(n, m^{\prime}\right) .
$$

$[-,-]$ being $S$-bilinear and $R$-balanced and $(-,-)$ being $R$-bilinear and $S$-balanced is equivalent to having bimodule maps $\varphi: N \otimes_{S} M \rightarrow R$ and $\psi: M \otimes_{R} N \rightarrow S$, given by

$$
\varphi(n \otimes m)=(n, m) \quad \text { and } \quad \psi(m \otimes n)=[m, n]
$$

so that the associativity conditions above read

$$
\varphi(n \otimes m) n^{\prime}=n \psi\left(m \otimes n^{\prime}\right) \quad \text { and } \quad \psi(m \otimes n) m^{\prime}=m \varphi\left(n \otimes m^{\prime}\right) .
$$

A Morita context is a sextuple $(R, S, N, M, \varphi, \psi)$ satisfying the conditions given above. The associated ring is called the Morita ring of the context. By abuse of notation we will write $(R, S, N, M)$ instead of $(R, S, N, M, \varphi, \psi)$ and will suppose $R, S, N, M$ contained in the Morita ring associated to the context. The Morita context will be called surjective if the maps $\varphi$ and $\psi$ are both surjective.

In classical Morita theory, it is shown that two rings with identity $R$ and $S$ are Morita equivalent (i.e., $R$-mod and $S$-mod are equivalent categories) if and only if there exists a surjective Morita context $(R, S, N, M, \varphi, \psi)$. 
The approach to Morita theory for rings without identity by means of Morita contexts appears in a number of papers (see [20] and the references therein) in which many consequences are obtained from the existence of a Morita context for two rings $R$ and $S$.

For an idempotent ring $R$ we denote by $R$-Mod the full subcategory of the category of all left $R$-modules whose objects are the "unital" nondegenerate modules. Here, a left $R$-module $M$ is said to be unital if $M=R M$, and $M$ is said to be nondegenerate if, for $m \in M, R m=0$ implies $m=0$. Note that, if $R$ has an identity, then $R$-Mod is the usual category of left $R$-modules $R$-mod.

It is shown in [25, Theorem] that, if $R$ and $S$ are arbitrary rings having a surjective Morita context, then the categories $R$-Mod and $S$-Mod are equivalent. It is proved in [20, Proposition 2.3] that the converse implication holds for idempotent rings.

Given two idempotent rings $R$ and $S$, we will say that they are Morita equivalent if the respective full subcategories of unital nondegenerate modules over $R$ and $S$ are equivalent.

The following result can be found in [20] (see Proposition 2.5 and Theorem 2.7).

Theorem 2.2. Let $R$ and $S$ be two idempotent rings. Then the categories $R-M o d$ and $S-M o d$ are equivalent if and only if there exists a surjective Morita context $(R, S, M, N)$.

The socles of Morita equivalent semiprime idempotent rings are closely related as we will see next. The proofs of the following results are largely based on the concept of local algebra at an element that we proceed to introduce.

For a ring $R$ and an element $x \in R$, the local ring of $R$ at $x$ (denoted $R_{x}$ ) is defined to be the ring $x R x$, with the sum inherited from $R$, and product given by $x a x \cdot x b x=x a x b x$. The use of local rings at elements allows to overcome the lack of a unit element in the original ring, and to translate problems from a non-unital context to the unital one. See [21] for an equivalent definition and information about the exchange of properties between a ring and its local rings at elements. In particular, if $e$ is an idempotent in the ring $R$, then the local ring of $R$ at $e$ is just the corner $e R e$.

If $R$ is a semiprime ring, then the sum of all its minimal left ideals coincides with the sum of all its minimal right ideals. This sum is called the socle of $R$ and will be denoted by $\operatorname{Soc}(R)$. When the ring has no minimal one-sided ideals, it is said that $R$ has zero socle.

As the next lemma shows, taking local rings at elements and considering the socle are commuting operations. 
Lemma 2.3. For a semiprime ring $R$ and an element $x \in R$, we have $\operatorname{Soc}\left(R_{x}\right)=(\operatorname{Soc}(R))_{x}$.

Proof. Note that for every element $x \in R$, being $R$ semiprime implies $R_{x}$ is semiprime too (apply [21, Proposition 2.1 (i)]), hence it makes sense to consider the socle of the local ring at that element.

Show first $\operatorname{Soc}\left(R_{x}\right) \subseteq(\operatorname{Soc}(R))_{x}$. If $x a x \in \operatorname{Soc}\left(R_{x}\right)$, by [21, Proposition $2.1(\mathrm{v})]$, xax $\in \operatorname{Soc}(R)$. As the socle is a von Neumann regular ring, there exists $y \in R$ such that $x a x=(x a x) y(x a x)=(x a x) y(x a x) y(x a x)$; use that the socle is an ideal of the ring and that $x a x$ is in the socle of $R$ to obtain axyxaxyxa $\in \operatorname{Soc}(R)$, so that $x a x=x(\operatorname{axyxaxyxa}) x \in x(\operatorname{Soc}(R)) x$.

For the converse, consider $x a x \in x(\operatorname{Soc}(R)) x$, with $a \in \operatorname{Soc}(R)$. Apply again that the socle is an ideal to deduce that $x a x$ is in $\operatorname{Soc}(R)$. By $[21$, Proposition $2.1(\mathrm{v})]$ xax $\in \operatorname{Soc}\left(R_{x}\right)$, as wanted.

Theorem 2.4. Let $R$ and $S$ be two Morita equivalent semiprime idempotent rings. Then:

(1) $R$ has nonzero socle if and only if $S$ has nonzero socle.

(2) $R=\operatorname{Soc}(R)$ if and only if $S=\operatorname{Soc}(S)$.

Proof. We start by setting several notation and results that will be used to prove the statements.

Denote by $A$ the Morita ring associated to a surjective Morita context $(R, S, N, M)$, and identify $R, S, N$ and $M$, in the natural way, with subsets of $A$.

(i) Use Lemma 2.3 to settle that for any $x \in R$ we have: $(\operatorname{Soc}(R))_{x}=$ $\operatorname{Soc}\left(R_{x}\right)=\operatorname{Soc}\left(A_{x}\right)=(\operatorname{Soc}(A))_{x}$.

(ii) $\operatorname{Soc}(R)=\operatorname{Soc}(A) \cap R$ and $\operatorname{Soc}(S)=\operatorname{Soc}(A) \cap S$. This follows from (i) together with the fact that $R_{x}=A_{x}$ for every $x \in R$ and the fact that an element is in the socle of a ring if and only if the local ring at the element is an artinian ring (see [21, Proposition $1.2(\mathrm{v})]$ ).

(1). Take a nonzero element $x$ in $\operatorname{Soc}(R)$. By (ii), $x \in \operatorname{Soc}(A)$, and as the socle is an ideal, $M x N$, which is contained in $S$, is in the socle of $A$ too. We claim that $M x N$ is nonzero because otherwise $0=N M x N=R x R$, a contradiction since every element in the socle is von Neumann regular and $x$ is nonzero. Therefore we have $0 \neq M x N \subseteq \operatorname{Soc}(A) \cap S \subseteq \operatorname{Soc}(S)$.

It can be proved, in an analogous way, that $\operatorname{Soc}(S) \neq 0$ implies $\operatorname{Soc}(R) \neq 0$.

(2). If $R$ coincides with its socle, $R \subseteq \operatorname{Soc}(A)$. Then $S=M N=$ $M N M N=M R N \subseteq M(\operatorname{Soc}(A)) N \subseteq \operatorname{Soc}(A) \cap S=\operatorname{Soc}(S)$. 
The results above can be readily adapted to the Leavitt path algebra setting.

Corollary 2.5. Let $E$ be an arbitrary graph and $F$ any desingularization of E. Then

(1) $\operatorname{Soc}\left(L_{K}(E)\right) \neq 0$ if and only if $\operatorname{Soc}\left(L_{K}(F)\right) \neq 0$.

(2) $L_{K}(E)$ coincides with its socle if and only if $L_{K}(F)$ coincides with its socle.

Proof. Use first [3, Theorem 5.2] to get that $L_{K}(E)$ is Morita equivalent to $L_{K}(F)$. Now the proof is a straightforward consequence of Theorem 2.4 and the fact that Leavitt path algebras for arbitrary graphs are rings with local units (hence, idempotent rings), and also semiprime $[3$, Proposition 6.1].

The following result is a generalization of [12, Corollary 4.3] for arbitrary graphs.

Corollary 2.6. Let $E$ be an arbitrary graph, then $L_{K}(E)$ has nonzero socle if and only if $P_{l}(E) \neq \emptyset$.

Proof. Consider any desingularization $F$ of $E$. Apply Corollary 2.5 (1) to obtain that $\operatorname{Soc}\left(L_{K}(E)\right) \neq 0$ if and only if $\operatorname{Soc}\left(L_{K}(F)\right) \neq 0$. By the rowfinite case proved in [12, Corollary 4.3] we know that $\operatorname{Soc}\left(L_{K}(F)\right) \neq 0$ if and only if $P_{l}(F) \neq \emptyset$. Finally, use Proposition 2.1 (2) to get the result.

One of the main aims of the paper is the complete determination of the socle of a Leavitt path algebra of an arbitrary graph as the ideal generated by its set of line points. Unfortunately, this description of the socle is unreachable via Morita equivalence (that is, by using a desingularization process). Among other things, because two Morita equivalent idempotent rings can have socles of different size. For example:

Example 2.7. Let $R:=R C F M(K)$ be the ring of infinite matrices with entries in a field $K$, and finite rows and columns. Consider in $R$ the idempotent $e_{11}$ (defined as the matrix having 1 in place $(1,1)$ and zero elsewhere), and denote $f:=1-e \in R$. Then ( $e R e, f R f, f R e, e R f)$ is a surjective Morita context for the two idempotent rings $e R e$ and $f R f$, and while $e R e$ is finite dimensional (in fact, it is isomorphic to the base field), $f R f$ is not.

In the upcoming sections of the paper we will use specifically-adapted methods in order to achieve our main goal: the description of the socle. 


\section{Action of the multiplication algebra of $L_{K}(E)$}

The action of the multiplication algebra of a Leavitt path algebra on the algebra itself has proved to be a powerful tool that has allowed researchers to shorten the proofs of some results in this theory. Recall that for a not necessarily associative $K$-algebra $A$, and fixed $x, y \in A$, the left and right multiplication operators $L_{x}, R_{y}: A \rightarrow A$ are defined by $L_{x}(y):=x y$ and $R_{y}(x):=x y$. Denoting by $\operatorname{End}_{K}(A)$ the $K$-algebra of $K$-linear maps $f: A \rightarrow A$, the multiplication algebra of $A$ (denoted $\mathfrak{M}(A)$ ) is the subalgebra of $\operatorname{End}_{K}(A)$ generated by the unit and all left and right multiplication operators $L_{a}, R_{a}: A \rightarrow A$. There is a natural action of $\mathfrak{M}(A)$ on $A$ such that $A$ is an $\mathfrak{M}(A)$-module whose submodules are just the ideals of $A$. This is given by $\mathfrak{M}(A) \times A \longrightarrow A$, where $f \cdot a:=f(a)$ for any $(f, a) \in \mathfrak{M}(A) \times A$. Given $x, y \in A$ we shall say that $x$ is linked to $y$ if there is some $f \in \mathfrak{M}(A)$ such that $y=f(x)$. This fact will be denoted by $x \vdash y$.

The result that follows was proved in [12, Proposition 3.1] for row-finite graphs. It states that any nonzero element in a Leavitt path algebra is linked to either a vertex or to a nonzero polynomial in a cycle with no exits. So it gives a full account of the action of $\mathfrak{M}\left(L_{K}(E)\right)$ on $L_{K}(E)$. This result proved to be very powerful as the main ingredient to show that the socle of a Leavitt path algebra of a row-finite graph is the ideal generated by the line points. The same proof given there can be used in the case of not necessarily row-finite graphs.

Proposition 3.1. Let $E$ be an arbitrary graph. Then, for every nonzero element $x \in L_{K}(E)$, there exist $\mu_{1}, \ldots, \mu_{r}, \nu_{1}, \ldots, \nu_{s} \in E^{0} \cup E^{1} \cup\left(E^{1}\right)^{*}$ such that:

(1) $\mu_{1} \ldots \mu_{r} x \nu_{1} \ldots \nu_{s}$ is a nonzero element in $K v$, for some $v \in E^{0}$, or

(2) there exist a vertex $w \in E^{0}$ and a cycle without exits $c$ based at $w$ such that $\mu_{1} \ldots \mu_{r} x \nu_{1} \ldots \nu_{s}$ is a nonzero element in $w L_{K}(E) w$.

Both cases are not mutually exclusive.

Corollary 3.2. For any nonzero $x \in L_{K}(E)$ we have $x \vdash v$ for some $v \in E^{0}$ or $x \vdash p\left(c, c^{*}\right)$ where $c$ is a cycle with no exits and $p$ a nonzero polynomial in $c$ and $c^{*}$.

Proof. Use Lemma 1.5 together with Proposition 3.1.

For any $K$-algebra $A$ the $\mathfrak{M}(A)$-submodules of $A$ are just the ideals of $A$ and the cyclic $\mathfrak{M}(A)$-submodules of $A$ are the ideals generated by one element (principal ideals in the sequel). So the previous corollary states that the 
nonzero principal ideals contain either vertices or nonzero elements of the form $p\left(c, c^{*}\right)$. Therefore, for graphs in which every cycle has an exit, each nonzero ideal contains a vertex. Now, [31, Corollary 6.10] can be obtained immediately from item (2) in the following result:

Corollary 3.3. Let $E$ be an arbitrary graph.

(1) Every $\mathbb{Z}$-graded nonzero ideal of $L_{K}(E)$ contains a vertex.

(2) Suppose that E satisfies Condition (L). Then every nonzero ideal of $L_{K}(E)$ contains a vertex.

Proof. The second assertion has been proved above. So assume that $I$ is a graded ideal of $L_{K}(E)$ which contains no vertices. Let $0 \neq x \in I$ and use Corollary 3.2 to find elements $y, z \in L_{K}(E)$ such that $y x z=\sum_{i=-m}^{n} k_{i} c^{i} \neq 0$. But $I$ being a graded ideal implies that every summand is in $I$. In particular, for $t \in\{-m, \ldots, n\}$ such that $k_{t} c^{t} \neq 0$ we have $0 \neq\left(k_{t}\right)^{-1} c^{-t} k_{t} c^{t}=w \in I$, which is absurd.

It was shown in [3, Proposition 6.1] that $L_{K}(E)$ is a semiprime algebra for an arbitrary graph. The proof required the use of the desingularization process. Here, we can give an element-wise proof by using Proposition 3.1.

Proposition 3.4. Let $E$ be an arbitrary graph. Then $L_{K}(E)$ is semiprime.

Proof. Take a nonzero ideal $I$ such that $I^{2}=0$. If $I$ contains a vertex we are done. Otherwise there is a nonzero element $p\left(c, c^{*}\right) \in I$ by Corollary 3.2. If we consider the (nonzero) coefficient of maximum degree in $c$ and write $p\left(c, c^{*}\right)^{2}=0$ we immediately see that this scalar must be zero, a contradiction.

To illustrate the power of Proposition 3.1 we can see how it reduces considerably in length the proofs given in [31] of the so-called Uniqueness Theorems. These are [31, Theorem 4.6] (Graded Uniqueness Theorem) and [31, Theorem 6.8] (Cuntz-Krieger Uniqueness Theorem).

Theorem 3.5. Let $E$ be an arbitrary graph, and let $L_{K}(E)$ be the associated Leavitt path algebra.

(1) Graded Uniqueness Theorem.

If $A$ is a $\mathbb{Z}$-graded ring and $\pi: L_{K}(E) \rightarrow A$ is a graded ring homomorphism with $\pi(v) \neq 0$ for every vertex $v \in E^{0}$, then $\pi$ is injective.

(2) Cuntz-Krieger Uniqueness Theorem.

Suppose that $E$ satisfies Condition (L). If $\pi: L_{K}(E) \rightarrow A$ is a ring homomorphism with $\pi(v) \neq 0$ for every vertex $v \in E^{0}$, then $\pi$ is injective. 
Proof. In both cases, the kernel of the ring homomorphism $\pi$ is an algebra ideal (a graded ideal in the first one). By Corollary 3.3, $\operatorname{Ker}(\pi)$ must be zero because otherwise it would contain a vertex (apply (1) in Corollary 3.3 to (1) and (2) to the other case), which is not possible by the hypotheses.

In [31], Tomforde used the previous theorems in order to prove that, for the field of complex numbers $\mathbb{C}$, the Leavitt path algebra $L_{\mathbb{C}}(E)$ could be embedded in the graph $\mathrm{C}^{*}$-algebra $C^{*}(E)$ via a homomorphism $\phi: L_{\mathbb{C}}(E) \rightarrow$ $C^{*}(E)$ sending the generators of $L_{\mathbb{C}}(E)$ to the generators of $C^{*}(E)$. As he noted, such a homomorphism is well-defined by the universal property of $L_{\mathbb{C}}(E)$ and is injective, as can be shown precisely by applying the Graded Uniqueness Theorem. Here, we can use this embedding $\phi$ to get that the socle of the Leavitt path algebra of an arbitrary graph $E$ is always contained in the socle of the graph $\mathrm{C}^{*}$-algebra of $E$ (but may not be equal), as is shown in the next result. The counterexample contained in the following proposition was communicated to the authors by Pere Ara.

Proposition 3.6. Let $E$ be an arbitrary graph. Then

$$
\operatorname{Soc}\left(L_{\mathbb{C}}(E)\right) \subseteq \operatorname{Soc}\left(C^{*}(E)\right)
$$

Moreover, there exists a row-finite graph E such that the inclusion is proper.

Proof. As explained in the previous paragraph, we can use Theorem 3.5 (1) and the ideas of [31, Proof of Theorem 7.3] to obtain that $L_{\mathbb{C}}(E)$ is isomorphic to a dense ${ }^{*}$-subalgebra $\mathcal{A}$ of $C^{*}(E)$.

Consider a minimal idempotent $e \in \mathcal{A}$. We will show that $e$ is a minimal idempotent in $C^{*}(E)$ as well. It suffices to show that $e C^{*}(E) e$ is a division ring. Take a nonzero element $x \in e C^{*}(E) e$. Because $\mathcal{A}$ is a dense *-subalgebra of $C^{*}(E)$, there exists a sequence $\left\{x_{n}\right\}_{n=1}^{\infty} \subseteq \mathcal{A}$ such that $x=\lim \left(e x_{n} e\right)$. Suppose that $n$ is such that the element $e x_{n} e \in e \mathcal{A} e$ is nonzero. Since $e \mathcal{A} e$ is a division ring, there exists $y_{n} \in e \mathcal{A} e$ such that $e x_{n} e y_{n}=y_{n} e x_{n} e=\left.1\right|_{e \mathcal{A} e}=e$. As $x \neq 0$, there exists $m$ such that $e x_{n} e \neq 0$ for every $n \geq m$. Define $y_{n}=0$ for every $n<m$ and $y=\lim y_{n}$. Then $x y=\lim \left(e x_{n} e\right) y_{n}=\lim e=e=\left.1\right|_{e C^{*}(E) e}$ and analogously $y x=\left.1\right|_{e C^{*}(E) e}$. This proves our claim.

Recall that $\operatorname{Soc}(R)$ is the two-sided ideal generated by the minimal idempotents of a ring $R$. Denote by $\mathcal{I}$ the set of the minimal idempotents in $\mathcal{A}$, and by $\mathcal{I}^{*}$ the set of minimal idempotents in $C^{*}(E)$. Then:

$$
\operatorname{Soc}(\mathcal{A})=\sum_{e \in \mathcal{I}} \mathcal{A} e \mathcal{A} \subseteq \sum_{e \in \mathcal{I}} C^{*}(E) e C^{*}(E) \subseteq \sum_{e \in \mathcal{I}^{*}} C^{*}(E) e C^{*}(E)=\operatorname{Soc}\left(C^{*}(E)\right)
$$


To show that the inclusion might be proper we consider the row-finite graph $E$ given by

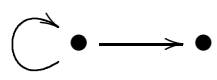

In [29], Siles Molina showed that the Leavitt path algebra of this graph is the algebraic Toeplitz algebra $T=\mathbb{C}\langle x, y \mid x y=1\rangle$, for which it is known that $\operatorname{Soc}(T)=\mathbb{M}_{\infty}(\mathbb{C})$. However, the completion of $T$ is the analytic Toeplitz algebra, whose socle is the algebra of finite rank operators, which strictly contains $\mathbb{M}_{\infty}(\mathbb{C})$ (matrices of countable size with only a finite number of nonzero entries).

In spite of the power of the aforementioned Uniqueness Theorems, one may encounter different situations in which neither set of hypotheses in Theorem 3.5 are satisfied. This happens, for instance, in the proof of [3, Proposition 5.1]. Here, the authors showed that for any arbitrary graph $E$, and $F$ any of its desingularizations, the Leavitt path algebra $L_{K}(E)$ is isomorphic to a subalgebra of $L_{K}(F)$. In proving this result, they built a homomorphism $\phi$ from $L_{K}(E)$ to $L_{K}(F)$ and needed to show its injectivity. In this situation, neither $E$ satisfied Condition (L) nor $\phi$ was a graded homomorphism (because the desingularization process might enlarge some paths but not all of them), so the Uniqueness Theorems could not be applied. The key point of the proof they gave was just that the image of a certain cycle without exits was again a cycle, possibly with more edges than the original one. This idea appears in the main result of [30], i.e., Theorem 1.2. The hypotheses in these cases were less general than the ones in the following generalization of the Cuntz-Krieger Uniqueness Theorem.

Theorem 3.7. Let $E$ be an arbitrary graph, $A$ a graded $K$-algebra and $\pi: L_{K}(E) \rightarrow A$ a ring homomorphism with $\pi(v) \neq 0$, for every vertex $v \in E^{0}$, which maps each cycle without exits to a non-nilpotent homogeneous element of nonzero degree. Then $\pi$ is injective.

Proof. Note that the kernel of $\pi$ is an algebra ideal of $L_{K}(E)$ which does not contain vertices. If $\operatorname{Ker}(\pi)$ is nonzero, by Corollary 3.2 it contains a nonzero element $p\left(c, c^{*}\right)$, where $p$ is a polynomial and $c$ is a cycle without exits. By the hypothesis $\pi(c)=h \neq 0$ is a homogeneous element of degree $r \neq 0$, thus $0=\pi\left(p\left(c, c^{*}\right)\right)=p\left(h, h^{*}\right)$. Since $h$ is not nilpotent, then the coefficients of the polynomial $p\left(c, c^{*}\right)$ are all zero, a contradiction.

Finally we show how to use Proposition 3.1 to simplify the proof on the characterization of simple Leavitt path algebras (see [3, Theorem 3.1]).

Corollary 3.8. Let $E$ be an arbitrary graph. Then $L_{K}(E)$ is simple if and only if $E$ satisfies Condition (L) and the only hereditary and saturated subsets of $E^{0}$ are the trivial ones. 
Proof. If $L_{K}(E)$ is simple, both conditions on the graph $E$ are proved in [3, Theorem 3.1]. For the converse take into account that Condition (L) implies that any nonzero element in $L_{K}(E)$ is linked to a vertex (see Proposition 3.3). Thus, there is a vertex in any nonzero ideal $I$ of $L_{K}(E)$. But on the other hand $\emptyset \neq I \cap E^{0}$ is hereditary and saturated ([3, Lemma 2.3]), therefore it coincides with $E^{0}$ and so $I=L_{K}(E)$.

\section{Minimal left ideals}

Minimal left ideals are the building pieces of the socle of a semiprime ring. Clearly enough, in order to be able to compute $\operatorname{Soc}\left(L_{K}(E)\right)$, it would be wise to collect as much information as possible on the structure of these ideals. Hence, the aim of this section is to find necessary and sufficient conditions so that a principal left ideal is minimal.

As a first step, we will find necessary and sufficient conditions on a vertex so that the left ideal it generates turns out to be minimal. We recall some notions introduced and results proved in [12] for row-finite graphs, which will be also useful in the context of arbitrary graphs.

We say that a path $\mu$ contains no bifurcations if the set $\mu^{0} \backslash\{r(\mu)\}$ contains no bifurcations, that is, if none of the vertices of the path $\mu$, except perhaps $r(\mu)$, is a bifurcation.

The following two results are valid verbatim for arbitrary graphs because the use of relation (4) in their proofs is limited to the case of vertices $v$ without bifurcations (and therefore finite-emitters).

Lemma 4.1. [12, Lemma 2.2] Let $E$ be an arbitrary graph and let $u, v$ be in $E^{0}$, with $v \in T(u)$. If there is only one path joining $u$ with $v$ and it does not contain bifurcations, then $L_{K}(E) u \cong L_{K}(E) v$ as left $L_{K}(E)$-modules.

Note that the following proposition assumes that $u$ is a finite-emitter.

Proposition 4.2. [12, Proposition 2.3] Let $E$ be an arbitrary graph and $u$ a regular vertex with $s^{-1}(u)=\left\{f_{1}, \ldots, f_{n}\right\}$. Then $L_{K}(E) u=\bigoplus_{i=1}^{n} L_{K}(E) f_{i} f_{i}^{*}$. Furthermore, if $r\left(f_{i}\right) \neq r\left(f_{j}\right)$ for $i \neq j$ and $v_{i}:=r\left(f_{i}\right)$, then $L_{K}(E) u \cong$ $\bigoplus_{i=1}^{n} L_{K}(E) v_{i}$.

The next result, however, requires a slight adaptation from its row-finite analog.

Lemma 4.3. Let $E$ be an arbitrary graph and let $u \in E^{0}$ be an infinite emitter. Then $\bigoplus_{i=1}^{\infty} L_{K}(E) f_{i} f_{i}^{*} \subsetneq L_{K}(E) u$, where $s^{-1}(u)=\left\{f_{i}\right\}_{i \in \mathbb{N}}$. In particular, $L_{K}(E) u$ is not a minimal left ideal. 
Proof. The inclusion $\bigoplus_{i=1}^{\infty} L_{K}(E) f_{i} f_{i}^{*} \subseteq L_{K}(E) u$ is clear. Suppose that $u \in \bigoplus_{i=1}^{\infty} L_{K}(E) f_{i} f_{i}^{*}$ and write $u=\sum_{j} \alpha_{j} g_{j} g_{j}^{*}$, where $g_{j} \in s^{-1}(u)$. Since $s^{-1}(u)$ is infinite, there exists $f \in s^{-1}(u)$ such that $f \neq g_{j}$ for all $j$. Then, $f=u f=\sum_{j} \alpha_{j} g_{j} g_{j}^{*} f=0$, a contradiction.

Recall that a left ideal $I$ of an algebra $A$ is said to be minimal if it is nonzero and the only left ideals of $A$ that it contains are 0 and $I$. From the results above we get an immediate consequence.

Corollary 4.4. Let $E$ be an arbitrary graph and $w \in E^{0}$. If $T(w)$ contains some bifurcation, then the left ideal $L_{K}(E) w$ is not minimal.

Thus we have found a first necessary condition for the minimality of the left ideal generated by a vertex. But, as in the row-finite case, there is a second condition, introduced in [12]. The proof given there holds also in our more general setting.

Proposition 4.5. [12, Proposition 2.5] Let $E$ be an arbitrary graph. If there is some closed path based at $u \in E^{0}$, then $L_{K}(E) u$ is not a minimal left ideal.

Thus using this proposition and Corollary 4.4 we conclude:

Proposition 4.6. Let $E$ be an arbitrary graph. Let $u$ be a vertex of the graph $E$ and suppose that the left ideal $L_{K}(E) u$ is minimal. Then $u \in P_{l}(E)$.

As we shall prove in what follows, this necessary condition turns out to be also sufficient. Following the reasoning given in [12, Proposition 2.7] but using Corollary 4.4 and Propositions 3.4 and 4.6 instead, we have:

Proposition 4.7. Let $E$ be an arbitrary graph. For any $u \in E^{0}$, the left ideal $L_{K}(E) u$ is minimal if and only if $u L_{K}(E) u=K u \cong K$.

Remark 4.8. For any $\operatorname{sink} u$, trivially $u L_{K}(E) u=K u \cong K$, and therefore the left ideal $L_{K}(E) u$ is minimal. Also, if $w$ is a vertex connected to a sink $u$ by a path without bifurcations, then we have that $L_{K}(E) w$ is a minimal left ideal because $L_{K}(E) w \cong L_{K}(E) u$ by Lemma 4.1.

Our task now is to show that the converse implication of Proposition 4.6 holds too. The proof of this fact strongly differs from that given in the row-finite setting, and this is so precisely because we lack the direct limit construction in which a great part of the proof for the row-finite case is based on. Our new approach is more combinatorial.

Before proceeding with this task, we need to establish several preliminary results. 
Proposition 4.9. Let $E$ be an arbitrary graph and $u \in P_{l}(E)$. Then every nonzero element of the form $f_{1} \cdots f_{k} g_{1}^{*} \cdots g_{p}^{*}$ with $r\left(g_{p}^{*}\right)=u$ and $s\left(f_{1}\right), r\left(f_{i}\right), r\left(g_{i}^{*}\right) \in T(u)$, is either the vertex $u$ or can be written as $g_{k+1}^{*} \cdots g_{p}^{*}$, with $1<k<p$.

Proof. We proceed by induction on the number of real edges $k$. If we have $f_{1} g_{1}^{*} \cdots g_{p}^{*}$, since $r\left(f_{1}\right)=s\left(g_{1}^{*}\right)=r\left(g_{1}\right)$ then $f_{1}=g_{1}$, and therefore $f_{1} g_{1}^{*} \cdots g_{p}^{*}$ $=g_{1} g_{1}^{*} \cdots g_{p}^{*}=g_{2}^{*} \cdots g_{p}^{*}$, by (4). Suppose the result is valid for $k-1$. Consider $f_{1} \cdots f_{k} g_{1}^{*} \cdots g_{p}^{*}$; by the induction hypothesis $f_{2} \cdots f_{k} g_{1}^{*} \cdots g_{p}^{*}=$ $g_{k}^{*} \cdots g_{p}^{*}$ so that $f_{1} \cdots f_{k} g_{1}^{*} \cdots g_{p}^{*}=f_{1} g_{k}^{*} \cdots g_{p}^{*}=g_{k+1}^{*} \cdots g_{p}^{*}$.

Lemma 4.10. Let $E$ be an arbitrary graph and let $\mu, \nu \in E^{*}$, with $l(\mu), l(\nu)$ $\geq 1, s(\mu)=s(\nu)$ and such that for every $u \in \mu^{0} \cup \nu^{0}$ there are neither bifurcations nor cycles at $u$. Then, $\mu \nu^{*} \neq 0$ implies $\mu \nu^{*}=s(\mu)$.

Proof. We prove it by induction on $l(\mu)+l(\nu)$. The base case is for $l(\mu)+l(\nu)=2$. In this case we have $\mu=f_{1}$ and $\nu=g_{1}$, with $s\left(f_{1}\right)=$ $r\left(g_{1}^{*}\right)=s\left(g_{1}\right)$, and since we have no bifurcations at $s\left(f_{1}\right)$, necessarily $f_{1}=g_{1}$ and moreover, by $(4)$ we get $f_{1} g_{1}^{*}=s\left(f_{1}\right)$.

Let us suppose the result holds for the cases with $l(\mu)+l(\nu)<n$, and prove it for $l(\mu)+l(\nu)=n$. Write $\mu=f_{1} \ldots f_{r}$ and $\nu=g_{s} \ldots g_{1}$. Note that by the hypothesis we have $r, s \geq 1$. Now, since $\mu \nu^{*} \neq 0$, then

$$
\text { (§) } f_{2} \ldots f_{r} g_{1}^{*} \ldots g_{s-1}^{*} \neq 0 \text {. }
$$

In this situation we have three possibilities:

If $r=1$, then again having no bifurcations at $s\left(f_{1}\right)$ implies that $f_{1}=g_{s}$, and by $(\S)$ we get that $r\left(f_{r}\right)=s\left(g_{1}^{*}\right)$, that is, $s\left(g_{1}^{*}\right)=r\left(g_{s}\right)=s\left(g_{s}^{*}\right)=r\left(g_{s-1}^{*}\right)$. In other words, $g_{s-1} \ldots g_{1}$ is a closed path based at $r\left(f_{1}\right)$, and therefore there exists some cycle based at this same vertex, contradicting our assumption. If $s=1$ we may proceed analogously. Finally, for the case $r, s>1$ we are allowed to apply the induction hypothesis on $(\S)$ with the paths $\mu^{\prime}=$ $f_{2} \ldots f_{r}$ and $\nu^{\prime}=g_{s-1} \ldots g_{1}$ which of course verify that $s\left(\mu^{\prime}\right)=s\left(\nu^{\prime}\right)$. Thus, $\mu^{\prime}\left(\nu^{\prime}\right)^{*}=s\left(\mu^{\prime}\right)$ and consequently $\mu \nu^{*}=f_{1} s\left(\mu^{\prime}\right) g_{s}^{*}=f_{1} f_{1}^{*}=s\left(f_{1}\right)$, again by using (4) and the fact that there are no bifurcations at $s\left(f_{1}\right)$.

We would like to determine the different types of monomials we might encounter in $L_{K}(E) u$ when $u \in P_{l}(E)$. In order to do this, we make the following definitions. Define $L$ to be the set of edges $f \in E^{1}$ so that $s(f), r(f) \in T(u)$. It is clear that the sources of edges in $E^{1} \backslash L$ are not in $T(u)$ because there are not bifurcations at any vertex of $T(u)$. Therefore, for all paths $\mu=f_{1} \cdots f_{k}$ with $f_{i} \in L$ and $\tau=g_{1} \cdots g_{p}$ with $g_{j} \notin L$, we have $\mu \tau=0$ because $r(\mu) \in T(u)$ but $s(\tau) \notin T(u)$. Moreover, the graph $T=\left(T(u), L,\left.r\right|_{T(u)},\left.s\right|_{T(u)}\right)$ is a line graph (that is, a graph without bifurcations which has only $u$ as a source). 
Proposition 4.11. Let $E$ be an arbitrary graph and $u \in P_{l}(E)$. The monomials generating $L_{K}(E) u$ as a $K$-vector space are of the following types:

(1) $u$.

(2) $f_{1} \cdots f_{k}$, with $r\left(f_{k}\right)=u$ and $s\left(f_{1}\right) \neq u$.

(3) $f_{1} \cdots f_{k} g_{1}^{*} \cdots g_{p}^{*}$ with $s\left(f_{1}\right) \neq u, r\left(g_{p}^{*}\right)=u, f_{i} \notin L$ and $g_{i} \in L$.

(4) $g_{1}^{*} \cdots g_{p}^{*}$ with $r\left(g_{p}^{*}\right)=u, s\left(g_{1}^{*}\right) \neq u$ and $g_{i} \in L$.

Proof. Consider first a monomial in real edges. Then it is necessarily of one the types (1) or (2). Take next a monomial of mixed type with real and ghost edges. Then it must be of the form $f_{1} \cdots f_{k} g_{1}^{*} \cdots g_{p}^{*}$ with $r\left(g_{p}^{*}\right)=u$. In case $s\left(f_{1}\right)=u$, applying Lemma 4.10 we fall again in case (1). So we can proceed supposing $s\left(f_{1}\right) \neq u$. If for some $i$ we have $f_{i} \in L$, then $i=k$ or $f_{i+1}, \ldots, f_{k} \in L$. Since $f_{i} \cdots f_{k} g_{1}^{*} \cdots g_{p}^{*}$ is nonzero and all the edges are in $L$ (and also $r\left(g_{p}^{*}\right)=u$ ) we have $f_{i} \cdots f_{k} g_{1}^{*} \cdots g_{p}^{*}=g_{j}^{*} \cdots g_{p}^{*}$ for some $j$ so that $1 \leq j \leq p$, by Proposition 4.9 .

In a similar fashion we can proceed with the elements of type (4) to see that $g_{i} \in L$.

We have now all the technical ingredients in hand to prove that the necessary condition for a principal left ideal generated by a vertex to be minimal, given in Proposition 4.6, is also sufficient.

Theorem 4.12. Let $E$ be an arbitrary graph and $u \in E^{0}$. Then $L_{K}(E) u$ is a minimal left ideal if and only if $u \in P_{l}(E)$.

Proof. Let $u \in E^{0}$ such that $L_{K}(E) u$ is minimal. Then $u \in P_{l}(E)$ by Proposition 4.6.

Now we prove the converse. Take $u \in P_{l}(E)$ and $0 \neq z \in L_{K}(E) u$. We will show that $u \in L_{K}(E) z$. By Proposition 4.11 we may write $z=$ $z_{1}+z_{2}+z_{3}+z_{4}$, where $z_{i}$ is a linear combination of monomials of type (i) in Proposition 4.11. We distinguish four cases.

Case 1: $z_{1} \neq 0$.

In this situation $z_{1}=k u$ for some $0 \neq k \in K$, so $u=k^{-1} u z_{1}=k^{-1} u z \in$ $L_{K}(E) z$.

Case 2: $z_{1}=0$ and $z_{4} \neq 0$.

Let $k t_{1}^{*} \cdots t_{l}^{*}$ be a nonzero monomial in $z_{4}$ with $l$ minimal. For every monomial $f_{1} \cdots f_{k}$ of type (2) appearing in $z_{2}$ we have that $u t_{l} \cdots t_{1} f_{1} \cdots f_{k}=0$ since $C P(u)=\emptyset$. Pick a nonzero monomial $f_{1} \cdots f_{k} g_{1}^{*} \cdots g_{p}^{*}$ of type $(3)$ of $z_{3}$. Then $u t_{l} \cdots t_{1} f_{1} \cdots f_{k} g_{1}^{*} \cdots g_{p}^{*}=0$ because $f_{i} \notin L$ and $t_{i} \in L$. Moreover, if 
we consider $g_{1}^{*} \cdots g_{p}^{*}$, a monomial of type (4) appearing in $z_{4}$, different from $t_{1}^{*} \cdots t_{l}^{*}$, we have that $u t_{l} \cdots t_{1} g_{1}^{*} \cdots g_{p}^{*}=0$ because otherwise there would exist a closed path based at $u$ (observe that since $l$ is minimal, we necessarily have $l<p)$. This shows that $u=k^{-1} u t_{l} \cdots t_{1} z \in L_{K}(E) z$.

Case 3: $z_{1}, z_{4}=0$ and $z_{2} \neq 0$.

Choose $k t_{1} \ldots t_{l}$, a nonzero monomial in $z_{2}$ with $l$ maximal. Note that $t_{l}^{*} \cdots t_{1}^{*} z_{2}=k u$ because for a nonzero monomial $f_{1} \cdots f_{r}$ different from $t_{1} \cdots t_{l}$ appearing in $z_{2}$ we have that $t_{l}^{*} \cdots t_{1}^{*} f_{1} \cdots f_{r} \neq 0$ would imply $l>r$ and consequently $C P(u) \neq \emptyset$, a contradiction.

Now, choose $f_{1} \cdots f_{r} g_{1}^{*} \cdots g_{s}^{*}$, a monomial of type (3) and consider the element $x=t_{l}^{*} \cdots t_{1}^{*} f_{1} \cdots f_{r} g_{1}^{*} \cdots g_{s}^{*}$. Distinguish the following three situations. First, if $r<l$, then $x=t_{l}^{*} \cdots t_{r+1}^{*} g_{1}^{*} \cdots g_{s}^{*}=0$ since $C P(u)=\emptyset$. Second, if $r=l$ then $x=u x=u g_{1}^{*} \cdots g_{s}^{*}=0$ as $C P(u)=\emptyset$. Finally, if $r>l$ then $x=t_{l}^{*} \cdots t_{1}^{*} f_{1} \cdots f_{l} f_{l+1} \cdots f_{r} g_{1}^{*} \cdots g_{s}^{*}=u f_{l+1} \cdots f_{r} g_{1}^{*} \cdots g_{s}^{*}$, and this would imply $f_{l+1} \in L$, a contradiction.

This proves that $t_{l}^{*} \cdots t_{1}^{*} z_{3}=0$ so that $u=k^{-1} t_{l}^{*} \cdots t_{1}^{*} z_{2}=k^{-1} t_{l}^{*} \cdots t_{1}^{*} z \in$ $L_{K}(E) z$.

Case $4: z_{1}, z_{2}, z_{4}=0$ and $z_{3} \neq 0$.

Choose $k t_{1} \cdots t_{l} h_{1}^{*} \cdots h_{m}^{*}$, a nonzero monomial in $z_{3}$ with $l$ minimal. Now we have two possibilities:

(i) There is some summand $f_{1} \cdots f_{r} g_{1}^{*} \cdots g_{s}^{*}$ of $z_{3}$ such that $f_{1} \cdots f_{r} \neq$ $t_{1} \cdots t_{l}$. If $r=l$ then $t_{l}^{*} \cdots t_{1}^{*} f_{1} \cdots f_{r} g_{1}^{*} \cdots g_{s}^{*}=0$, whereas if $r>l$, we would get that $s\left(f_{l+1}\right) \in T(u)$ so that $f_{l+1} \in L$, which is impossible.

(ii) $z_{3}$ is the monomial $k t_{1} \cdots t_{l} h_{1}^{*} \cdots h_{m}^{*}$.

Hence, in any case, $t_{l}^{*} \cdots t_{1}^{*} z$ is an element which is under the conditions in Case 2, and therefore $u \in L_{K}(E) t_{l}^{*} \cdots t_{1}^{*} z \subseteq L_{K}(E) z$.

We close this section with the result that states that minimal left ideals are generated by line points. The proof of this fact follows the same sketch that the proof of [12, Theorem 3.4], now using Lemma 1.5, Proposition 3.1 and Theorem 4.12 instead of their row-finite analogs.

Theorem 4.13. Let $E$ be an arbitrary graph and let $x$ be in $L_{K}(E)$ such that $L_{K}(E) x$ is a minimal left ideal. Then, there exists a vertex $v \in P_{l}(E)$ such that $L_{K}(E) x$ is isomorphic (as a left $L_{K}(E)$-module) to $L_{K}(E) v$. 


\section{The socle of a Leavitt path algebra}

Leavitt path algebras are semiprime by Proposition 3.4. This implies, in particular, that their left and right socles agree, which enables us to speak of the socle without distinguishing sides. However, it is more convenient for us to work with left ideals, hence we will obtain the socle as the sum of all minimal left ideals.

Recall that a homogeneous component of the socle is the sum of all minimal left ideals which are isomorphic among themselves. Each homogeneous component is also a (two-sided) ideal and the sum of all of them is the socle. Having characterized in the previous section the minimal left ideals, we can apply these results to characterize $\operatorname{Soc}\left(L_{K}(E)\right)$. First of all we would like to give a generating set of vertices of the socle as a two-sided ideal.

Proposition 5.1. For an arbitrary graph $E$ we have that

$$
\sum_{u \in P_{l}(E)} L_{K}(E) u \subseteq \operatorname{Soc}\left(L_{K}(E)\right) .
$$

The reverse containment does not hold in general.

Proof. Use Theorem 4.12 to show that for any $u \in P_{l}(E)$, the left ideal $L_{K}(E) u$ is minimal and therefore it is contained in the socle.

The reverse containment is not true in general as shows the example given in [12, Proposition 4.1].

As in the case of a row-finite graph, the socle of a Leavitt path algebra $L_{K}(E)$ is generated as a two-sided ideal by $P_{l}(E)$, the set of line points. To prove this, we can follow the steps in the proof of [12, Theorem 4.2] but using Propositions 3.4, 4.13 and 5.1 rather than their row-finite versions, jointly with the fact that the ideal generated by a subset $H$ of $E^{0}$ agrees with the ideal generated by the hereditary saturated closure of $H$ (see the first assertion of [10, Lemma 2.1]).

Theorem 5.2. Let $E$ be an arbitrary graph. Then

$$
\operatorname{Soc}\left(L_{K}(E)\right)=I\left(P_{l}(E)\right)=I(H),
$$

where $H$ is the hereditary and saturated closure of $P_{l}(E)$.

This result has an immediate but useful corollary.

Corollary 5.3. For an arbitrary graph $E$, the Leavitt path algebra $L_{K}(E)$ has nonzero socle if and only if $P_{l}(E) \neq \emptyset$.

If $R$ is a ring, we let $\mathbb{M}_{\infty}(R)$ denote the ring of matrices of countable size over $R$ with only a finite number of nonzero entries. 
Examples 5.4. By using these results, we can compute the socle of some Leavitt path algebras of not necessarily row-finite graphs.

1. Consider the infinite edges graph $E_{\infty}$ given by

$$
\bullet \stackrel{(\infty)}{\longrightarrow} \bullet w
$$

Then, by [3, Lemma 1.1], $L_{K}\left(E_{\infty}\right) \cong \mathbb{M}_{\infty}(K) \vee K$, where the latter denotes the unitization of $\mathbb{M}_{\infty}(K)$. Thus Theorem 5.2 gives another way to show that $\operatorname{Soc}\left(\mathbb{M}_{\infty}(K) \vee K\right)=\mathbb{M}_{\infty}(K)$ via the Leavitt path algebra approach because

$\operatorname{Soc}\left(\mathbb{M}_{\infty}(K) \vee K\right)=\operatorname{Soc}\left(L_{K}\left(E_{\infty}\right)\right)=I\left(P_{l}\left(E_{\infty}\right)\right)=I(\{w\})=\mathbb{M}_{\infty}(K)$,

where the last equality can be obtained by using the isomorphism defined in [3, Lemma 1.1].

2. Take the infinite clock graph $C_{\infty}$

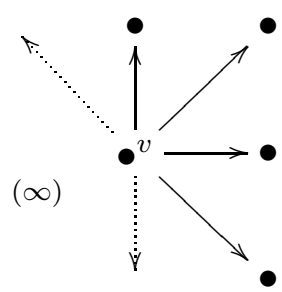

By [3, Lemma 1.2] we know that

$$
L_{K}\left(C_{\infty}\right) \cong \bigoplus_{i=1}^{\infty} \mathbb{M}_{2}(K) \oplus K I_{22},
$$

where $I_{22}$ is the element in $\prod_{i=1}^{\infty} \mathbb{M}_{2}(K)$ given by $I_{22}=\prod_{i=1}^{\infty} E_{22}$, and $E_{22}$ is the standard (2,2)-matrix unit in $\mathbb{M}_{2}(K)$. Thus, using again Theorem 5.2 and the isomorphism given in [3, Lemma 1.2] we get

$$
\begin{aligned}
\operatorname{Soc}\left(\bigoplus_{i=1}^{\infty} \mathbb{M}_{2}(K) \oplus K I_{22}\right) & =\operatorname{Soc}\left(L_{K}\left(C_{\infty}\right)\right)=I\left(P_{l}\left(C_{\infty}\right)\right) \\
& =I\left(C_{\infty}^{0} \backslash\{v\}\right)=\bigoplus_{i=1}^{\infty} \mathbb{M}_{2}(K) .
\end{aligned}
$$

The next corollary is a generalization of [12, Corollary 4.4]. Recall that $L_{K}(1, \infty)$ is the Leavitt path algebra of the infinite rose graph $R_{\infty}$

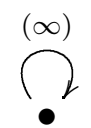

considered in [3, Examples 3.1 (ii)]. 
Corollary 5.5. For all $m, n \in \mathbb{N} \cup\{\infty\}$,

$$
\operatorname{Soc}\left(\mathbb{M}_{m}\left(L_{K}(1, n)\right)\right)=0 \text {. }
$$

Proof. When both $m$ and $n$ are finite, we just apply [12, Corollary 4.4]. In other cases we consider the graph $E_{n}^{m}$ given by

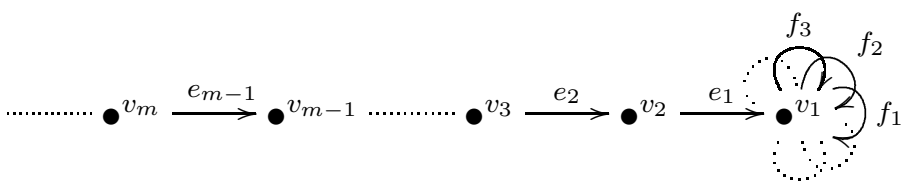

where if $m=\infty$ then we have an infinite number of edges and vertices in the line, and if $n=\infty$ then we have an infinite number of loops based at $v_{1}$. It is a tedious routine to check, with similar ideas to those of $[2$, Proposition $12]$, that $\mathbb{M}_{m}\left(L_{K}(1, n)\right) \cong L_{K}\left(E_{n}^{m}\right)$. This graph satisfies that $P_{l}\left(E_{n}^{m}\right)=\emptyset$, for every $m, n \in \mathbb{N} \cup\{\infty\}$ so that Corollary 5.3 yields the result.

We finish the paper by giving a structural characterization of the socle of a Leavitt path algebra $L_{K}(E)$ for an arbitrary graph $E$.

If the socle is nonzero, then we know that $\operatorname{Soc}\left(L_{K}(E)\right)=\oplus_{\alpha} C_{\alpha}$, where the $C_{\alpha}$ are the different homogeneous components which are simple $K$ algebras (agreeing with their socles).

The structure theorem of simple algebras which coincide with their socles states that any such algebra is isomorphic to an algebra $A=\mathfrak{F}_{\mathfrak{M}^{\prime}}(\mathfrak{M})$ (see $[23$, IV, $\S 8$, p. 74] for the definition). In the framework of this theory $\left(\mathfrak{M}, \mathfrak{M}^{\prime}\right)$ is a pair of dual vector spaces over a division $K$-algebra $\Delta$. These vector spaces come from the minimal left ideal $\mathfrak{M}=e A$ (so that $\mathfrak{M}^{\prime}=A e$ ) and $\Delta$ is the division $K$-algebra $\Delta=e A e$. Thus $\mathfrak{M}$ is a left $\Delta$-vector space and $\mathfrak{M}^{\prime}$ a right $\Delta$-vector space. Taking into account Proposition 4.7 we see that in our context $\Delta=K$ and the map $*: \mathfrak{M} \rightarrow \mathfrak{M}^{\prime}$ such that $e a \mapsto a^{*} e$ is an isomorphism of $K$-vector spaces. Hence $\operatorname{dim}(\mathfrak{M})=\operatorname{dim}\left(\mathfrak{M}^{\prime}\right) \leq \aleph_{0}$. Then, applying [23, IV,$\S 15$ Theorem 2, p. 89] in the infinite-dimensional case, each homogeneous component of $\operatorname{Soc}\left(L_{K}(E)\right)$ is isomorphic to $\mathbb{M}_{n}(K)$, where $n \in \mathbb{N} \cup\{\infty\}$.

Recall that a matricial algebra is a finite direct product of full matrix algebras over $K$, while a locally matricial algebra is a direct limit of matricial algebras. Now, Litoff's Theorem [23, IV, $\S 15$ Theorem 3, p. 90] implies that each homogeneous component of the socle is locally matricial over $K$ and so the socle itself is locally matricial over $K$. Thus we have proved the following 
Theorem 5.6. For any arbitrary graph $E$ the socle of the Leavitt path algebra $L_{K}(E)$ is zero or a locally matricial algebra and we have:

$$
\operatorname{Soc}\left(L_{K}(E)\right)=\bigoplus_{i \in I} \mathbb{M}_{n_{i}}(K)
$$

where $n_{i} \in \mathbb{N} \cup\{\infty\}$ and $I$ is a countable set.

\section{Acknowledgments}

The authors wish to thank Pere Ara for valuable comments and correspondence.

\section{References}

[1] Abrams, G. And Aranda Pino, G.: The Leavitt path algebra of a graph. J. Algebra 293 (2005), no. 2, 319-334.

[2] Abrams, G. and Aranda Pino, G.: Purely infinite simple Leavitt path algebras. J. Pure Appl. Algebra 207 (2006), no. 3, 553-563.

[3] Abrams, G. and Aranda Pino, G.: The Leavitt path algebras of arbitrary graphs. Houston J. Math. 34 (2008), no. 2, 423-442.

[4] Abrams, G., Aranda Pino, G., Perera, F. and Siles Molina, M.: Chain conditions for Leavitt path algebras. Forum Math 22 (2010), no. 1, 95-114.

[5] Abrams, G., Aranda Pino, G. and Siles Molina, M.: Finitedimensional Leavitt path algebras. J. Pure Appl. Algebra 209 (2007), no. 3, $753-762$.

[6] Abrams, G., Aranda Pino, G. and Siles Molina, M.: Locally finite Leavitt path algebras. Israel J. Math. 165 (2008), 329-348.

[7] Ara, P., González-Barroso, M. A., Goodearl, K. R. and ParDO, E.: Fractional skew monoid rings. J. Algebra 278 (2004), 104-126.

[8] Ara, P. And Pardo, E.: Stable rank for graph algebras. Proc. Amer. Math. Soc. 136 (2008), no. 7, 2375-2386.

[9] Ara, P., Moreno, M. A. and Pardo, E.: Nonstable K-Theory for graph algebras. Algebra Represent. Theory 10 (2007), no. 2, 157-178.

[10] Aranda Pino, G., Pardo, E. And Siles Molina, M.: Exchange Leavitt path algebras and stable rank. J. Algebra 305 (2006), no. 2, 912-936.

[11] Aranda Pino, G., Perera, F. and Siles Molina, M. (eds.): Graph algebras: bridging the gap between analysis and algebra. University of Málaga Press, Málaga, Spain, 2007. 
[12] Aranda Pino, G., Martín Barquero, D., Martín González, C. and Siles Molina, M.: The socle of a Leavitt path algebra. J. Pure Appl. Algebra 212 (2008), no. 3, 500-509.

[13] Aranda Pino, G. and Siles Molina, M.: The Maximal Graded Left Quotient Algebra of a Graded Algebra. Acta Math. Sinica, English Series 22 (2006), no. 1, 261-270.

[14] Bates, T., Hong, J.H., Raeburn, I. and Szymański, W.: The ideal structure of the $\mathrm{C}^{*}$-algebras of infinite graphs. Illinois J. Math. 46 (2002), no. $4,1159-1176$.

[15] Bates, T., Pask, D., Raeburn, I. and Szymański, W.: The C*algebras of row-finite graphs. New York J. Math. 6 (2000), 307-324.

[16] Cuntz, J.: Simple C*-algebras generated by isometries. Comm. Math. Physics 57 (1977), 173-185.

[17] Cuntz, J. And Krieger, W.: A class of $\mathrm{C}^{*}$-algebras and topological Markov chains. Invent. Math. 63 (1981), 25-40.

[18] Drinen, D. And Tomforde, M.: The $\mathrm{C}^{*}$-algebras of arbitrary graphs. Rocky Mountain J. Math 35 (2005), no. 1, 105-135.

[19] Fowler, N., Laca, M. and Raeburn, I.: The $C^{*}$-algebras of infinite graphs. Proc. Amer. Math. Soc. 8 (2000), 2319-2327.

[20] García, J. L. And Simón, J. J.: Morita equivalence for idempotent rings. J. Pure Appl. Algebra 76 (1991), 39-56.

[21] Gómez Lozano, M. and Siles Molina, M.: Quotient rings and Fountain-Gould left orders by the local approach. Acta Math. Hungar. 97 (2002), 287-301.

[22] Goodearl, K.R.: Leavitt path algebras and direct limits. Contemp. Math. 480 (2009), 165-187.

[23] Jacobson, N.: Structure of Rings. American Mathematical Society Colloquium Publications 37. American Mathematical Society, Providence, RI, 1968.

[24] Kumjian, A., Pask, D. And Raeburn, I.: Cuntz-Krieger algebras of directed graphs. Pacific J. Math. 184 (1998), no. 1, 161-174.

[25] Kyuno, S.: Equivalence of module categories. Math. J. Okayama Univ. 28 (1974), 147-150.

[26] Leaviti, W. G.: The module type of a ring. Trans. Amer. Math. Soc. 103 (1962), 113-130.

[27] Raeburn, I.: Graph algebras. CBMS Regional Conference Series in Mathematics 103. American Mathematical Society, Providence, RI, 2005.

[28] Raeburn, I. And Szymański, W.: Cuntz-Krieger algebras of infinite graphs and matrices. Trans. Amer. Math. Soc. 356 (2004), no. 1, 39-59.

[29] Siles Molina, M.: Algebras of quotients of path algebras. J. Algebra 319 (2008), no. 12, 329-348. 
[30] Szymański, W.: General Cuntz-Krieger uniqueness theorem. International J. Math 13 (2002), no. 5, 549-555.

[31] Tomforde, M.: Uniqueness theorems and ideal structure for Leavitt path algebras. J. Algebra 318 (2007), no. 1, 270-299.

Recibido: 16 de septiembre de 2008

Revisado: 7 de noviembre de 2008

$$
\begin{array}{r}
\text { Gonzalo Aranda Pino } \\
\text { Departamento de Álgebra, Geometría y Topología } \\
\text { Universidad de Málaga } \\
29071 \text { Málaga, Spain } \\
\text { g. aranda@uma.es } \\
\text { Dolores Martín Barquero } \\
\text { Departamento de Matemática Aplicada } \\
\text { Universidad de Málaga } \\
29071 \text { Málaga, Spain } \\
\text { dmartin@uma.es } \\
\text { Cándido Martín González } \\
\text { Universidad de Málaga } \\
29071 \text { Málaga, Spain } \\
\text { Departamento de Álgebra, Geometría y Topología } \\
\text { Mercedes Siles Molina } \\
\text { Geometría y Topología } \\
\text { Universidad de Málaga } \\
29071 \text { Málaga, Spain } \\
\text { msilesm@uma.es }
\end{array}
$$

The first author was supported by a Centre de Recerca Matemàtica Fellowship within the Research Programme "Discrete and Continuous Methods on Ring Theory". All authors were partially supported by the Spanish MEC and Fondos FEDER through projects MTM2004-06580-C02-02 and MTM2007-60333, and by the Junta de Andalucía and Fondos FEDER, jointly, through projects FQM-336, FQM-1215 and FQM-2467. This work has also been supported by the Spanish Ministry of Education and Science under project "Ingenio Mathematica (i-math)" No. CSD2006-00032 (Consolider-Ingenio 2010). 